\title{
Biomimetic Miniaturized Platform Able to Sustain Arrays of Liquid Droplets for High-Throughput Combinatorial Tests
}

\author{
Ana I. Neto, Clara R. Correia, Catarina A. Custódio and João F. Mano*
}

The development of high-throughput and combinatorial technologies is helping to speed up research that is applicable in many areas of chemistry, engineering, and biology. A new model is proposed for flat devices for the high-throughput screening of accelerated evaluations of multiplexed processes and reactions taking place in aqueous-based environments. Superhydrophobic (SH) biomimetic surfaces based on the so-called lotus effect are produced, onto which arrays of micro-indentations allow the fixing of liquid droplets, based on the rose-petal effect. The developed platforms are able to sustain arrays of quasi-spherical microdroplets, allowing the isolation and confinement of different combinations of substances and living cells. Distinct compartmentalized physical, chemical, and biological processes may take place and be monitored in each droplet. The devices permit the addition/ removal of liquid and mechanical stirring by adding magnetic microparticles into each droplet. By facing the chip downward, it is possible to produce arrays of cell spheroids developed by gravity in the suspended droplets, with the potential to be used as microtissues in drug screening tests.

provide parallel processing and monitoring of chemical and biological assays using low amounts of liquids. The conventional microtiter plate-based assays have moved away from the conventional 96-well format to the 384 well system, or even the 1536-well platform or high dense microfluidic devices. ${ }^{[8]}$ Tung et al. ${ }^{[9]}$ report a new technology enabling the culture of 384 spheroids in a hanging drop approach and in the same platform. However, the developed platform presents a single pattern and is fabricated by injection molding technology. The cost associated with the production of such innovative platforms can be extremely expensive due to the requirement of high precision equipment and expertise. Current developments in microfluidics, microarray systems, and microreactor devices are directed towards further reductions in sample volumes, increasing analytical throughput, and to

\section{Introduction}

The development of combinatorial methods can accelerate the scientific outcome, and thus the expansion of new materials and processes, when the effect of multiple variables is required. ${ }^{[1]}$ For example, high-throughput screening has been employed in the study of interactions between biomaterials, cells, and soluble factors, helping to identify the optimal conditions for particular biomedical applications. ${ }^{[2-4]}$ Microarrays enable the assembly of numerous cell-material or cell-cell combinations to analyze different parameters, including cell adhesion, proliferation, differentiation, and gene expression that can be monitored on a single microarray chip. ${ }^{[5-7]}$ Arraybased technologies have broad applications in drug discovery, microbiology, cell biology, and in many other non-biomedical fields. The high content and small scale of the microarrays

A. I. Neto, C. R. Correia, C. A. Custódio, Prof. J. F. Mano 3B's Research Group - Biomaterials

Biodegradables and Biomimetics

University of Minho, AvePark

4806-90, Taipas, Guimarães, Portugal

E-mail:jmano@dep.uminho.pt

A. I. Neto, C. R. Correia, C. A. Custódio, Prof. J. F. Mano

ICVS/3B's PT Government Associate Laboratory

Braga/Guimarães, Portugal

DOI: 10.1002/adfm.201400503 integrate the pre- and post-assay processing. Microarrays, such as DNA and protein quantification, have shown indispensable roles in genomic and proteomic research. ${ }^{[10]}$ However, in chemical microarrays, the evaluation of a large number of chemical structures and biological targets ${ }^{[11]}$ has been a slow process. The problem is associated with the fact that such microarrays do not allow the performance of individual analyses. In our group, we made advances in this field by creating an innovative platform for the individual dispensing of miniaturized biomaterials, cells, and culture media based on the use superhydrophobic (SH) surfaces patterned with wettable regions. ${ }^{[12-16]}$ The topography of these SH surfaces exhibit peculiar features like micro- and nanohierarchical roughness, leading to a low surface energy, mimicking $\mathrm{SH}$ surfaces found in nature, such as the Lotus leaf. We hypothesize that such SH substrates could be used to work with individual liquid volumes for a series of tests. Our main motivation is the handling of small amounts of liquid volumes, which is an important task in miniaturized bioanalytical and biomedical systems, since decreasing the size of the samples can reduce significantly the required time for the analysis and the associated costs.

In this work we propose the use of $\mathrm{SH}$ platforms to arrange quasi-spherical aqueous-based droplets with the capability to support and monitor a series of chemical and biological reactions on a lab-on-chip scale. Such platforms could have several advantages in comparison with the microarrays presented in the literature. The size and composition can be easily controlled 

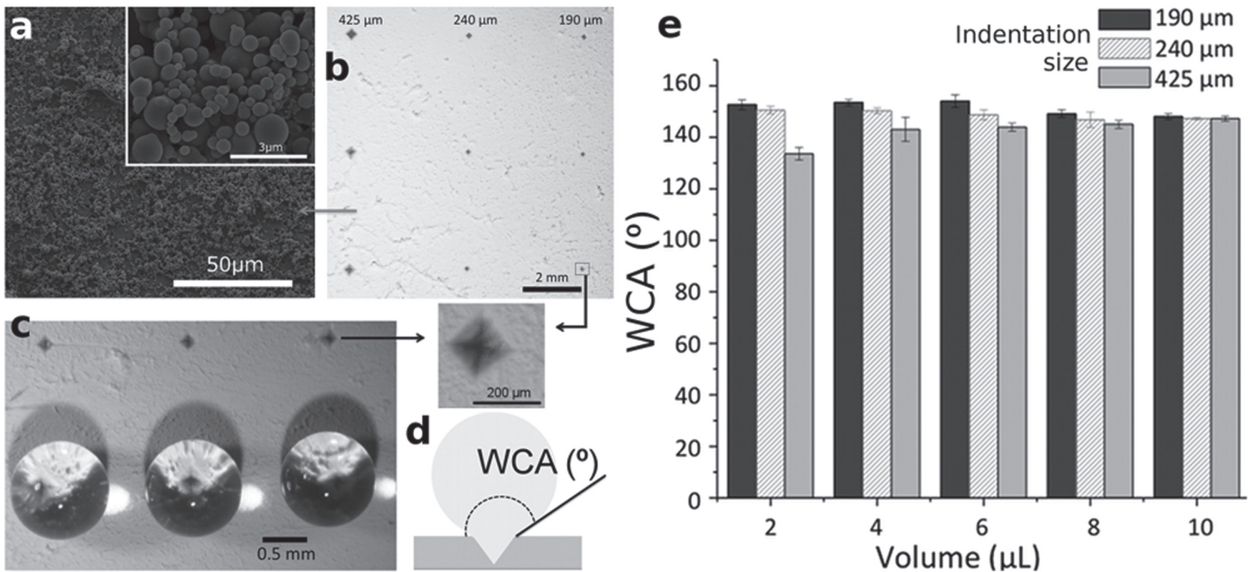

Figure 1. a) Microstructure of polystyrene SH surface observed by scanning electron microscope (SEM; two magnifications). b) Upper view of a SH platform patterned with micro-indentations to fix water droplets in the desired areas (c). d) Scheme of the water droplet position in the micro-indentations magnifying the effect of the penetration of the liquid in the mark. e) Water contact angle (WCA) measurement of water droplets with different volumes dispensed in the micro-indentations with different sizes.

in an individual form, and the processes in the liquid state occur with minimum contact with the solid substrate. Furthermore, the SH platform is solely physically modified polystyrene (PS), ${ }^{[17-19]}$ which guarantees the lack of cytotoxicity and the chemical stability of the platform.

We demonstrate that it is possible to add an agitation capability in the developed droplet-based microreactor using magnetic microspheres, enabling the creation of mechanical stresses inside the microliter-size droplets. The droplets are fixed in defined positions by the action of micro-indentations patterned with an array arrangement on the polystyrene SH surfaces. Different experiments are performed to demonstrate the suitability of the developed platform, including: (i) the efficacy of the mechanical agitation tested in a simple physical process, namely, the dissolution of salt crystals inside the droplets; (ii) the monitoring of a chemical reaction, namely the crosslinking of chitosan (CHT) with genipin with different concentrations of reagents; (iii) the evaluation of cell viability, in this case tested when cells are in contact with culture media at different $\mathrm{pHs}$, and; (iv) the evaluation of the cytotoxicity of an antitumor drug at different concentrations in cell spheroids. For the last example, we take advantage of the high positional stability and adhesiveness of the droplets that allow the inversion by $180^{\circ}$ of the SH platform. Therefore, cells initially suspended in the droplets give rise to spheroids, formed by gravity. The developed cell spheroids are used as microtissues for a drug delivery assay. The viability of the cells in the spheroids is assessed using image-based analysis tools upon adding doxorubicin, a drug used in cancer chemotherapy, in different concentrations.

\section{Results and Discussion}

\subsection{Preparation and Wettability Characterization of $\mathrm{SH}$ Patterned Chips}

Polystyrene SH surfaces were prepared via a phase-separation methodology, mimicking the extreme water repellency of lotus leavse by presenting a water contact angle (WCA) higher than $150^{\circ}$. This feature was achieved by the introduction of micro- and nanoroughness (Figure 1a), without further chemical modification of the polymeric structure. Such substrates present a similar topography previously observed in polystyrene $\mathrm{SH}$ substrates. ${ }^{[19]}$ The developed surfaces patterned with adhesive micro-indentations (Figure 1b) were used as support for arrays of aqueous solution-based droplets. Micro-indentations with different sizes were produced on the rough surface in order to fix liquid droplets, mimicking the effect of rose petals. In these surfaces, water droplets show a spherical shape, but they cannot roll off the surface even when the surface is turned upside down, ${ }^{[20-22]}$ because of the peculiar morphology characterized by micropapillae with cuticular folds on top. In contrast to the lotus surface where the air pockets formed between cell papillae provide low adhesion to droplets, the rose petal surface prevents air pocket formation and droplets penetrate into the cuticular folds by capillary forces. ${ }^{[23,24]}$ The micro- and nanostructures of the surface of the rose petals are larger than those found on the lotus leaves, allowing droplets to penetrate into the larger grooves of the petals, causing the Cassie impregnating wetting state. ${ }^{[23]}$

Water droplets placed on the micro-indentations exhibit a close spherical shape promoted by the extreme water repellency of the SH surface, but are fixed by the depression features. By producing multiple indentations over the $\mathrm{SH}$ surfaces one can generate attachment points to produce arrays of droplets with any geometrical disposition and distance between them (Figure 1c). The indentations created on low-surface-energy SH surfaces present some advantages when compared with highly adhesive SH surfaces, due to the possibility to confine and stabilize droplets only on the desired places with minimum contact areas between the droplet and the SH surface (Figure 1d).

The WCA was measured for droplets with distinct volumes placed over indentations of different sizes (Figure 1e). Small volumes of droplets in the micro-indentations present WCAs higher than $150^{\circ}$, showing that the smallest indentations on the SH surface are able to fix the droplets on the surface, without compromising the water repellency of the surface. 


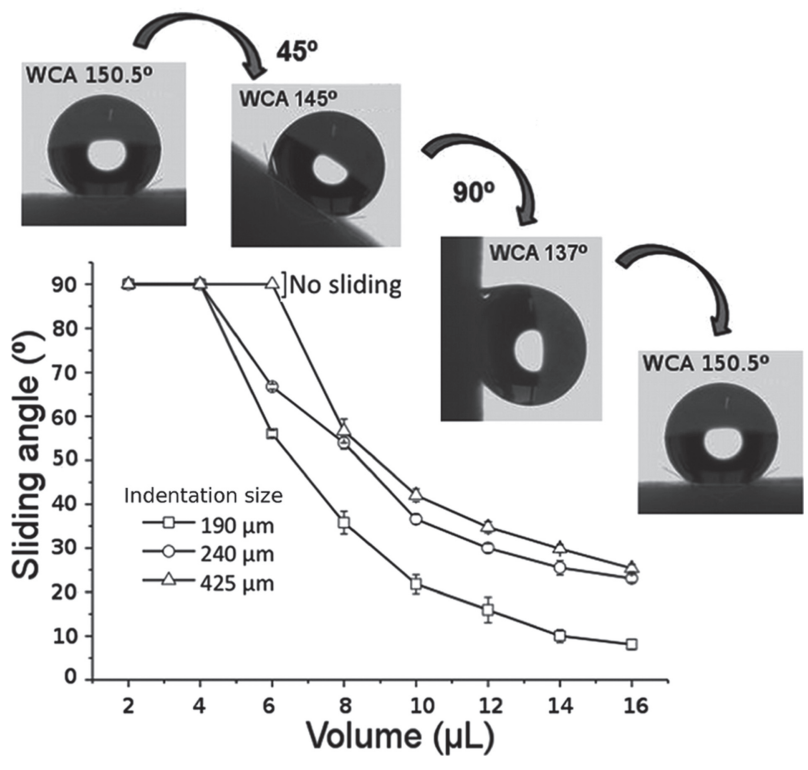

Figure 2. Sliding angles of droplets with different water volumes on patterned $\mathrm{SH}$ surfaces with different micro-indentation sizes (see legend). Representative images are shown of water droplets with $4 \mu \mathrm{L}$ deposited in micro-indentations with $190 \mu \mathrm{m}$ size in the surfaces with different tilt levels.

To assess the adhesive behavior of the indented points, the sliding angle was measured at different conditions of indentation sizes and droplet volumes (see Figure 2). The sliding angle is defined as the critical angle where a water droplet of a certain volume and weight begins to slide down the sloping surface. The results show that, when the liquid volume is lower than $4 \mu \mathrm{L}$, the water droplet cannot roll on nor be fixed on the surface for all micro-indentations sizes analyzed, even when the tilt of the surface is $90^{\circ}$. As expected, increasing the volume deposited in the micro-indentation decreases the sliding angles, since the capillary forces exert a lesser adhesion effect onto bigger droplets. Furthermore, the stability of the smallest droplets in the surfaces allows the WCA to remain unalterable, even after returning form a tilt of $90^{\circ}$ (Figure 2 - representative images).

We hypothesize that the ability to fix high stability arrays of controlled volumes of droplets, with minimum contact with the $\mathrm{SH}$ surfaces, could be useful in the design of innovative robust droplet-based microreactors.

\subsection{Microdroplet-based Microreactors Built on Patterned SH Surfaces}

Droplet-based microreactor technologies, such as microemulsions, array-based microreactors, or microfluidic systems, have attracted a great deal of interest since they enable the miniaturization of reactions. ${ }^{[25-28]}$ These systems have permitted significant advances in protein crystallization, enzymatic kinetics, cell encapsulation, drug delivery, and in other biochemical reactions ${ }^{[25]}$ using several methods such as electrowetting, ${ }^{[29]}$ dielectrophoresis, and magnetism. ${ }^{[30,31]}$ Shikida et al. ${ }^{[32]}$ developed a magnetic bead-cluster handling system in which the droplets are driven from one well to the next through a narrow

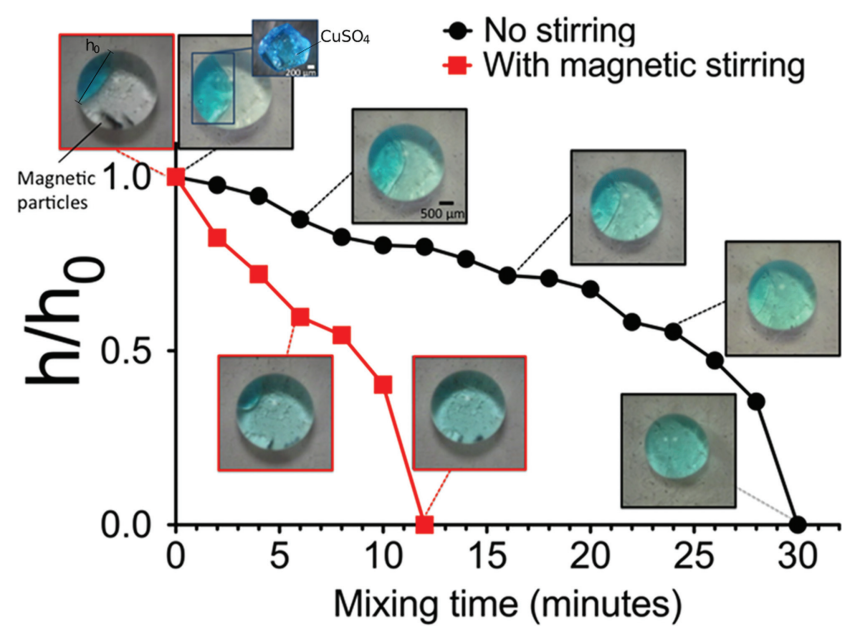

Figure 3. Miniaturized droplet-based reactor using water droplets with and without magnetic microparticles on a patterned SH surface. Crystals of copper sulphate were introduced inside the droplets at time 0 , and the platform, which contains droplets with magnetic particles inside, was placed over a magnetic stirrer. The dissolution of the crystal was monitored by following the size of the crystal $(h)$ compared with its initial size $\left(h_{0}\right)$.

connection by the displacement of an external magnet. Based on this technology, SH surfaces have been used to manipulate the movement of magnetic droplets with a permanent magnet underneath, ${ }^{[29,33]}$ with applicability in biotechnology and biomedicine, e.g., for high-throughput biochemical analysis and microassays. ${ }^{[34,35]}$ In this work, we present an alternative possibility for creating motion by activating the mobility of magnetic microparticles in fixed droplets to induce internal mechanical agitations. Video S1 in Supporting Information shows the possibility to create an active mixing approach based on magnetic microspheres incorporated inside microliter-size droplets.

Water droplets with and without magnetic microparticles were firstly deposited on a patterned $\mathrm{SH}$ surface. $\mathrm{CuSO}_{4}$ crystals were introduced inside the droplets at time 0 , and the platform was placed over a magnetic stirrer. The dissolution of the crystal was monitored by following the size of the crystal (h) with time compared to its initial size $\left(h_{0}\right)$ (Figure 3). For the droplets that contained magnetic beads, the crystal with an $\mathrm{h}_{0}$ of about $2 \mathrm{~mm}$ was completely dissolved after 12 min (see Video S2 in Supporting Information and Figure 3). On the other hand, without mechanical agitation, the dissolution time was double. Obviously, the agitation power may be easily controlled in the magnetic stirrer. This result shows that the usual agitation and mixing processes performed in many chemical and engineering processes may be miniaturized in microliter volumes using the proposed platforms. Such controlled agitation can be essential in many biochemical and biological processes, allowing the high throughput and effective study of complex processes on a lab-on-chip scale.

\subsection{Monitoring of a Crosslinking Reaction through Combinatorial Chemical Analysis}

One current problem in the development of chemical microarrays is that compounds with different structures and properties 
are traditionally screened in a solution phase, and individual reactions need to be isolated in well plates or tubes. There are several reasons to use droplets rather than well plates; namely, smaller volumes, higher speeds, and the isolation of the droplet content from the solid surface. Small volumes conserve expensive reagents and reduce the time required to assay extremely large libraries. Hydrogels have attracted a great interest in many fields, including biomedicine, due to their general high biocompatibility, ability to encapsulate cells, mechanical and structural properties similar to soft tissues, and their ability to be injected as a liquid which gels in situ using minimally invasive procedures. ${ }^{[36-38]}$ We have proposed SH-based chips to assess how cells interact with hydrogels in combinatorial studies, ${ }^{[14]}$ however, the evolution of the formation of the gels has never been followed in situ using microreactors.

Herein, we propose to analyze the formation of hydrogels on the developed platforms, starting with the deposition of combinations of liquid precursors in the form of droplets. We designed a combinatorial analysis to follow the crosslinking reaction of chitosan (CHT) with genipin, a natural crosslinking agent with low cytotoxicity. CHT hydrogels have been widely used in different biomedical applications. ${ }^{[39-41]}$ In particular, crosslinked CHT with genipin has been used in the preparation of elastics and hydrogels for cartilage substitutes, drug delivery systems, and the encapsulation of biological products. ${ }^{[42-45]}$ Results show that the resulting crosslinked complexes are not cytotoxic. ${ }^{[46]}$ The deposition of different droplet solutions containing different relative amounts of CHT and genipin was carried out in the developed microarray: distinct concentrations of $0.1,1,5,7.5$, and $10 \mathrm{mg} \mathrm{mL}^{-1}$ of CHT and genipin were analysed. The prepared solutions were dispensed in each spot with individual volumes of $10 \mu \mathrm{L}$. The same CHT solutions without genipin were used as a control. The experiment was performed at $40{ }^{\circ} \mathrm{C}$ under saturated humidity to avoid droplet evaporation. Figure $4 \mathrm{a}$ shows the time evolution of the droplets in the array during the reaction. The dark blue coloration that appears in the formed hydrogels is associated with the reaction between the amino groups of CHT and genipin. ${ }^{[44]}$ Independent calibration experiments, by reacting known amounts of glycine and genipin, permitted the production of standard curves relating the intensity of the blue color of the droplets and the extent of the reaction of the amine groups. An intensity map is shown in Figure $4 \mathrm{~b}$ for the array analyzed, where the evolution of the extent of reaction of the amine group of CHT is represented as function of the time for each combination analyzed.

When the concentration of CHT and genipin increased, more amino groups reacted and the speed of reaction increased, reaching values above $60 \%$ after $3 \mathrm{~h}$ of incubation. The system used could be extended to more complex combinations, for example, by including mixtures of different polymers able to react with genipin. For example, Silva et al. ${ }^{[42]}$ studied the crosslinking of the mixture of CHT and silk fibroin. The primary structure of fibroin chains contains a low percentage of amine-containing amino acids, such as lysine and arginine, that contribute to crosslinking sites upon reaction with genipin.

We envisage the possibility to monitor many other reactions occurring in aqueous media, provided that changes of color (even outside the visible spectrum) take place, such as addition or substitution reactions or redox processes.

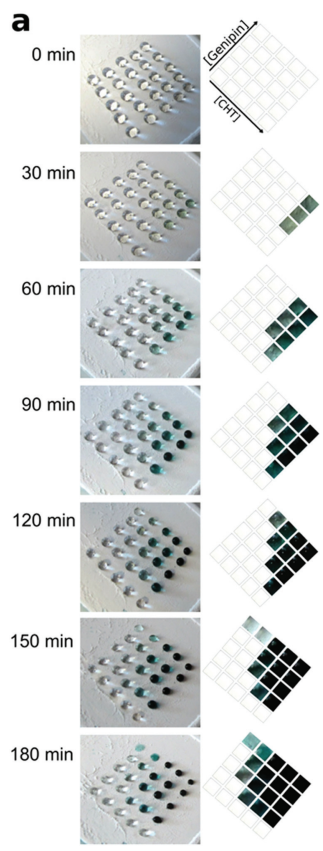

b

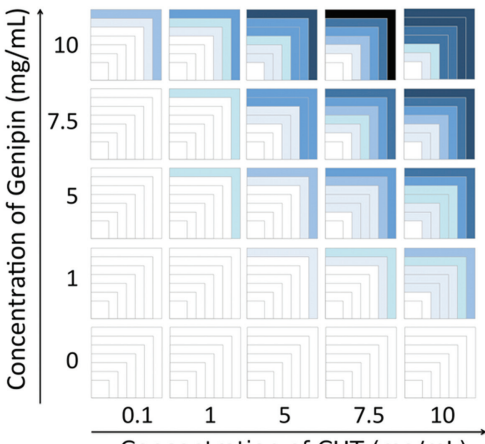

Concentration of $\mathrm{CHT}(\mathrm{mg} / \mathrm{mL})$
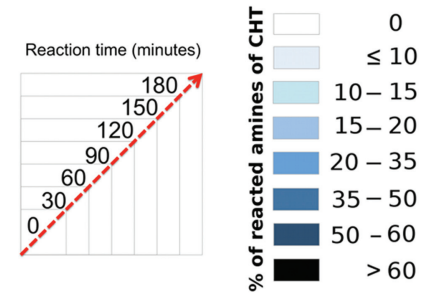

Figure 4. Combinatorial analysis following the reaction between $\mathrm{CHT}$ and genipin in arrays of droplets microreactors. a) Droplets with different concentrations of $\mathrm{CHT}$ and genipin were dispensed on a patterned $\mathrm{SH}$ surface. b) The intensity map displays the evolution of the crosslinking reaction between $\mathrm{CHT}$ and genipin for each concentration of reagents analysed. The blue intensity is correlated with the percentage of reacted amine groups of $\mathrm{CHT}$ at different time points.

\subsection{High-throughput Screening of Cell-based Assays}

Microarray platforms can address in vitro problems by creating distinct combinations of chemical/biochemical microenvironments to study cellular behavior. ${ }^{[47]}$ The advantage of the method proposed is that different biomaterials or even different types of cells can be easily deposited in each liquid spot with minimum contact with the supporting solid material. As a simple demonstration of this hypothesis, we evaluated the influence of the $\mathrm{pH}$ in the cellular medium on the viability of fibroblast-like cells.

It is known that the effect of the $\mathrm{pH}$ in cell culture media plays many critical roles in cell biological processes, such as viability, proliferation and apoptosis, ion transport, and multidrug resistance. ${ }^{[48]}$ Irregular $\mathrm{pH}$ values are usually associated with many diseases such as cancer. ${ }^{[49]}$ Tumor microenvironments are often acidic, with $\mathrm{pH}$ levels as low as 6.2. ${ }^{[50]}$ Inside the cells, the variations in the $\mathrm{pH}$ can denature proteins or activate enzymes that normally are inactive around neutral $\mathrm{pH}$. Knightom et al. ${ }^{[51]}$ showed that acidic conditions promote the angiogenesis of injured tissues. Figure 5 shows the viability of L929 cells suspended in culture media with different $\mathrm{pH}$ at different incubation times. Figure $5 \mathrm{a}$ shows the corresponding live/dead cell staining, obtained by the direct observation of the array of droplets dispensed in the platform (Figure 5b). As expected, and according to the literature, ${ }^{[52-54]}$ the fluorescence images confirmed that the favorable $\mathrm{pH}$ for cells to survive is between 6 and 8. In very acid and very alkaline media, all cells died before $5 \mathrm{~min}$. High-throughput image analysis of the 


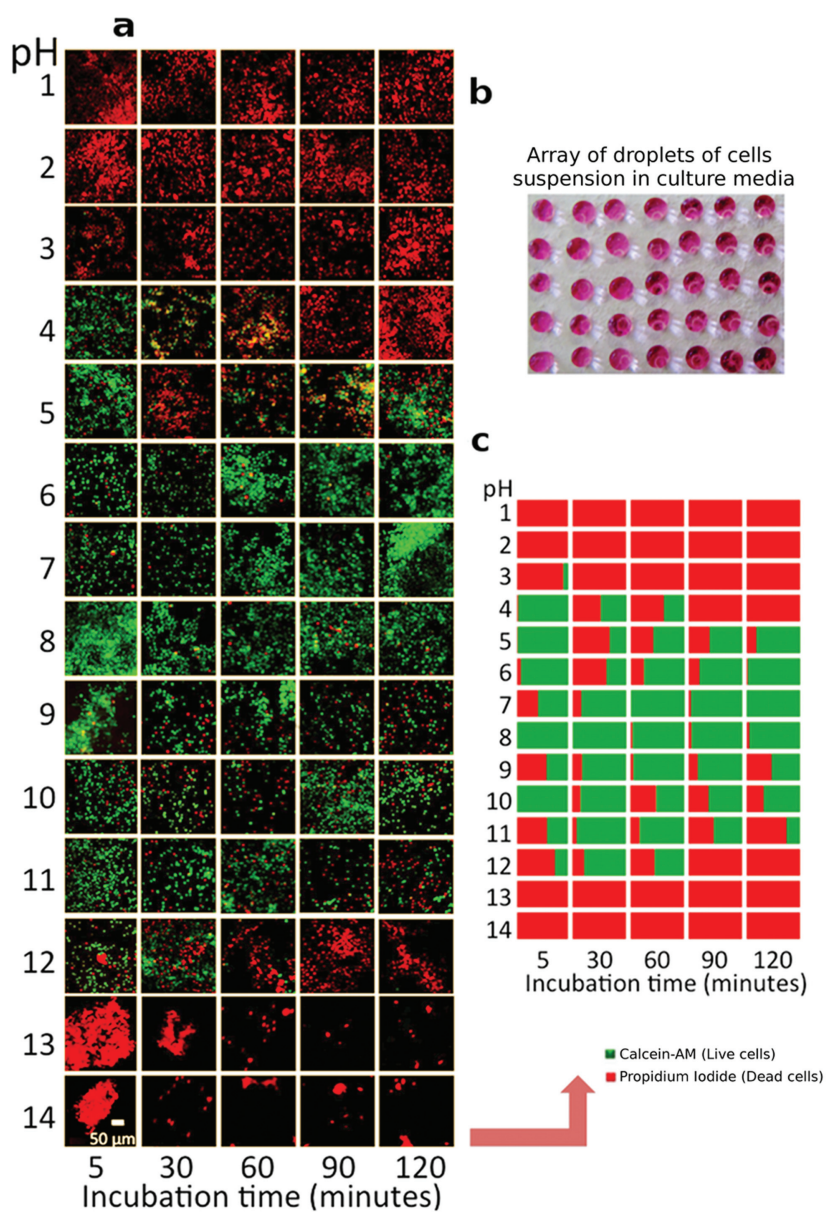

Figure 5. a) Fluorescence microscope images of the microarray composed of culture medium droplets at different $\mathrm{pH}$ values (vertical axis), containing $1 \times 10^{3}$ L929 cells/droplet, taken at different incubation times (horizontal axis). b) Representative top-view image of the microarray where $10 \mathrm{~mL}$ of solution of individual cells suspension were dispensed in patterned SH surfaces. c) Intensity map for the relative amount of live (green)/dead (red) cells per spot corresponding to the same microarray tested with different $\mathrm{pHs}$ and culture times.

micrographs permitted an intensity map of the values of the percentage of live/dead cells of the individual conditions to be built (Figure 5c), in which green and red colors represent the relative amount of living and dead cells. The results suggest that the proposed droplet-array chip may potentially be used to follow the cellular behavior of non-adherent cells, any cells in suspension, or to investigate the effect of the surrounding atmosphere.

Most cell-based assays are currently performed on monolayer cultures (2D substrates) ${ }^{[13]}$ or array plates, ${ }^{[9,55,56]}$ where cells adhere over a solid substrate, spread, and form stress fibers between focal adhesions. The use of microarrays for combinatorial analysis presents several advantages: high stability, good control with a homogeneous cell environment, and facilitated microscope analysis and medium changes. However, in native tissues, many types of cells interact with other cells and with an extracellular matrix (ECM) within 3D structures. The cell-cell and cell-ECM interactions provide mechanical and biochemical cues that can influence cellular functions and differentiation.

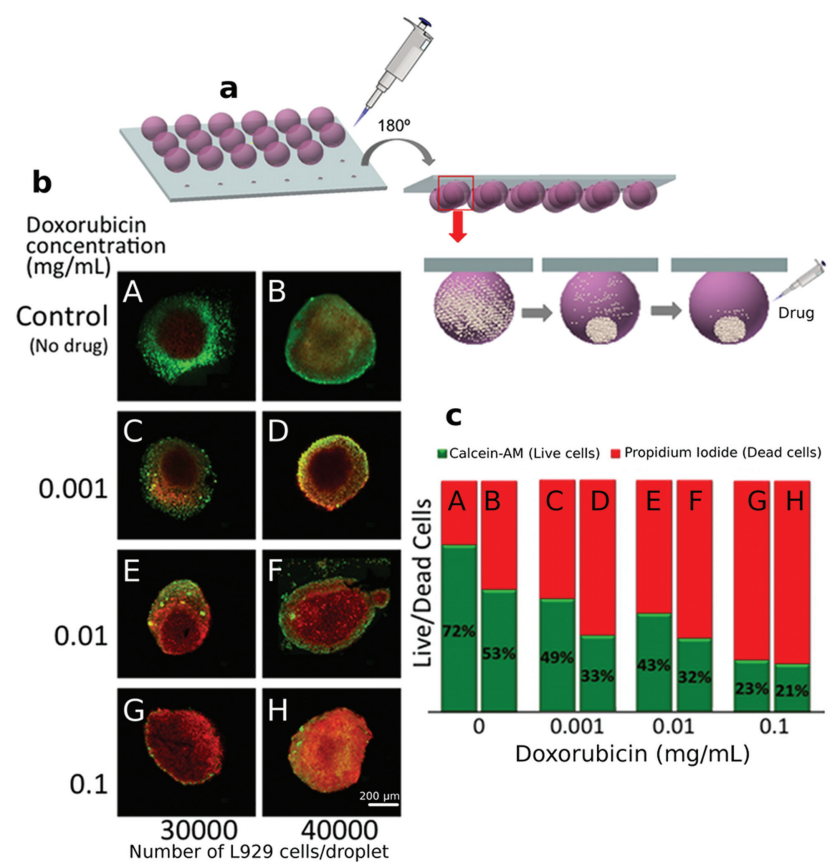

Figure 6. a) Scheme of SH surfaces patterned with micro-indentations able to suspend arrays of droplets containing cells; upon $24 \mathrm{~h}$, spheroids are formed and drug-screening tests may be performed on the individual droplets. b) Fluorescent images of L929 spheroids obtained from a confocal microscope $24 \mathrm{~h}$ after the addition of various concentrations of doxorubicin. c) Percentage of live (green)/dead (red) cells per spot in the different conditions shown in (b).

Herein, we propose a new process for the formation of arrays of 3D cell agglomerates to be used in drug screening. In the developed platform, the cells are maintained in suspension, confined within a miniature liquid cage surrounded essentially by the gaseous environment of the device. The micro-indentations produced on the SH surfaces promote the fixation of the droplets over the highly water-repellent surfaces, even when the surface and cell suspension droplets are turned upside down. Therefore, it is possible to produce an array of microliter volume droplets with an inverse configuration that could be useful for highthroughput monitoring. Extending the screening of cells in suspension of the last example, we employed the new arrangement to test the behavior of cells in an aggregated form (see Figure 6).

Commonly used methods to produce uniform cellular spheroids include: the hanging drop culture, microfluidic spheroid formation, non-adherent micropatterned surfaces, and culture in microwell plates. ${ }^{[56-61]}$ With the proposed platform it is possible to produce arrays of sedimented spheroids and test them in the same platform (see the scheme in Figure 6a). Under this configuration, the cells in the suspended droplets give rise to spheroids formed by gravity. In this case, the cells do not have any contact with a solid substrate, and the clusters of cells cultured in this environment allow cell-cell interactions to dominate over cell-substrate interactions. The gravitational force applied to the cells in the hanging drop suspended cultures caused sedimentation, and aggregates became visible after $24 \mathrm{~h}$.

The platform used in this work presents several advantages compared to conventional methods used for spheroid formation: 
the use of microsize droplets for cell growth assays allows a drastic reduction of the volume in which the cells are grown; the use of SH surfaces, mimicking the effect of rose petals, permits a minimum contact of the liquid to the surface, avoiding any interferences such as protein adsorption or the release of molecules from the platform to the liquid media; cell culture media can be changed easily without destroying the microtissues; drugs or other molecules can be added at any time, and if precautions are taken, the mechanical impact in the spheroids is reduced; the platform is compatible with or easily adapted to many analysis techniques, such as inverted confocal microscopes, in which droplets of cell suspensions remain inverted on the SH surface; high-throughput combinatorial analysis may be performed in situ using different cell densities or drug concentrations. For the proof-of-concept, droplets containing fibroblasts-like cells with two densities (30 000 and 40000 cells/ droplet) were dispensed on the inverted platform in order to promote the spheroids formation. After $24 \mathrm{~h}$, different amounts of doxorubicin were introduced into the droplets to assess the dose-dependent response of the formed tumor spheroids to this anticancer drug. The viability of the cells in the spheroids was measured after $24 \mathrm{~h}$ using live/dead staining images by confocal microscopy (Figure 6b). Images were selected out of the confocal stack to avoid overlapping of the same cells between images. Figure $6 c$ shows the resulting analysis of the fraction of live and dead cells in the different conditions. As expected, with the increase of the doxorubicin concentration, cell viability decreased in both types of spheroids. The percentage of living cells is higher in the smaller spheroids. This should be due to the fact that assessing nutrients and the release of waste is less efficient in the larger and denser spheroids, giving rise to more extensive cell death. The images of the Figure $6 \mathrm{~b}$ clearly show that dead cells are mostly accumulated in the inner regions of the spheroids.

\section{Conclusions}

A new concept of substrates for anchoring aqueous-based microliter droplets was developed based on the use of superhydrophobic flat substrates with controlled positional adhesion. Such microarray chips are proposed to be used as a new low-cost toolbox for high-throughput analyses in aqueousbased media. The platforms permit the screening of physical, chemical, and biological processes involving a combination of distinct elements and conditions, confined in spherical liquid minireactors. Events taking place in both homogeneous and heterogeneous environments can be monitored inside the fixed droplets onto which relevant manipulations can be made including: mechanical agitations, mixing, adding/removal of liquids and molecules or positioning the droplets at any angle relative to the platform. Besides following more conventional chemical reactions, the developed supports may also have potential to be used in biology or biomedical applications, including in the field of tissue engineering/regenerative medicine, biochemical and biological reactions, diagnosis, analytical analysis, drug discovery, and drug delivery monitoring.

\section{Experimental Section}

Fabrication of Patterned SH Surfaces: Smooth polystyrene (PS) sheets, purchased from Goodfellow, UK, with $0.19 \mathrm{~mm}$ of thickness, ref. ST311190/1, were modified into SH substrates using a one-step phase-separation methodology under ambient condition. The surface modification was produced by dissolving $70 \% \mathrm{v} / \mathrm{v}$ solution of PS (Styrolution PS $158 \mathrm{~K}$ ) in tetrahydrofuran (THF, from Sigma-Aldrich) solvent for approx. $2 \mathrm{~h}$ to form a pre-solution. Then, $1.4 \mathrm{~mL}$ of ethanol, from Panreac, was added into $2 \mathrm{~mL}$ of PS pre-solution and the mixed solution was stirred. A necessary volume to cover the PS substrate with this mixture was dipped onto a cleaned PS smooth sheet. After several seconds in air, the substrate with the mixture was immersed in ethanol for $1 \mathrm{~min}$ in order to force the polymer precipitation, leading to the formation of a rough and porous topography at both the nanoand microscale levels. Afterwards the surface was dried at ambient temperature.

Micro-indentations over the SH surfaces were produced using a microhardeness tester equipment (Leica VMHT 30) applying loads of $2942 \mathrm{mN}, 4903 \mathrm{mN}$ and $19610 \mathrm{mN}$ with a dwell time of $10 \mathrm{~s}$. A sharp rigid Vickers diamond pyramid indenter (included angle $\alpha=136^{\circ}$ ) penetrated into the surface with a constant load during a complete loading-unloading cycle producing individual indentations. To produce the pedestals able to sustain arrays of droplets, the indentations were placed in the surface at a distance of $5 \mathrm{~mm}$ from each other, with a square configuration.

The wettability of the smooth and polystyrene $\mathrm{SH}$ surfaces was assessed by contact angle measurements. Static water contact angle (WCA) measurements were carried out using an OCA15+ goniometer (DataPhysics, Germany) using the sessile drop method. Distilled water $(4 \mu \mathrm{L})$ was dropped on the surfaces and pictures were taken after stabilization of the water drop. The adhesion of droplets with different volumes in the micro-indentations with different sizes of the $\mathrm{SH}$ surfaces were also studied: the sliding WCA of droplets with different volumes was analysed in each micro-indentations by sloping $\mathrm{SH}$ surfaces until $90^{\circ}$.

The surface morphology of the SH samples was analysed by scanning electron microscopy, SEM (Leica Cambridge, UK).

Preparation of Microreactor Based on Patterned SH Surfaces: Microindentations with $190 \mu \mathrm{m}$ of size were produced in the SH surface using an indentation force of $2942 \mathrm{mN}$. Magnetic beads $\left(\mathrm{Fe}_{3} \mathrm{O}_{4}\right.$, diameter ranging from 5-50 $\mu \mathrm{m}$ ) were suspended and stirred until homogeneity in water with a concentration of $10 \mathrm{mg} \mathrm{mL}^{-1}$. A $10 \mu \mathrm{L}$ water droplet with magnetic microspheres impregnated was deposited over a microindented SH surface using a microdispenser. The droplet suspension was subjected to a magnetic field by placing a magnetic stirrer (Fisher Scientific) with variable speed below the SH surface. The experience started with an increasing speed until $100 \mathrm{rpm}$ allowing that the magnetic microspheres form chain-like structure under the influence of the imposed magnetic field. A cooper sulphate crystal was then incorporated into the droplet. Images and movies of the droplets during the dissolution of the crystal were obtained using a Stereo Microscope + Lamp Schott KL 200 (model Stemi 1000 PG-HITEC, Zeiss) with 2.5X magnification, equipped with a digital camera with $5 \times$ optical zoom.

Chemically Crosslinked Hydrogels: Water-soluble highly purified chitosan, $\mathrm{CHT}$ (Protasan UP CL 213, viscosity $107 \mathrm{mPa} . \mathrm{s}$, molecular weight $\mathrm{Mw}=2.7 \times 10^{5} \mathrm{~g} \mathrm{~mol}^{-1}, 83 \%$ degree of deacetylation) was purchased from NovaMatrix. Genipin was purchased from Wako chemicals (ref no. 078-03021). Solutions of CHT were prepared using $1 \% \mathrm{v} / \mathrm{v}$ of acetic acid at concentrations of $0.1 \mathrm{mg} \mathrm{mL}^{-1}, 1 \mathrm{mg} \mathrm{mL}^{-1}, 5 \mathrm{mg} \mathrm{mL}^{-1}, 7.5 \mathrm{mg} \mathrm{mL}^{-1}$ and $10 \mathrm{mg} \mathrm{mL}^{-1}$. Genipin solutions were prepared in ultra pure water at concentrations of $1 \mathrm{mg} \mathrm{mL}^{-1}, 5 \mathrm{mg} \mathrm{mL}^{-1}, 7.5 \mathrm{mg} \mathrm{mL}^{-1}$ and $10 \mathrm{mg} \mathrm{mL}^{-1}$. Both materials were mixed using the same quantity of the different concentrations leading to 20 different combinations. $\mathrm{CHT}$ solutions with the same concentrations without genipin were used as control.

The solutions were dropped in the chips prepared using the $\mathrm{SH}$ surfaces patterned with micro-indentations (190 $\mu \mathrm{m}$ size). The polymeric 
solutions were dispensed over the micro-indentations in a drop-bydrop logic in individual volumes of $10 \mu \mathrm{L}$. Each droplet separated by $5 \mathrm{~mm}$, represents a different combination of $\mathrm{CHT}$ and genipin. After dispensing the solutions, the $\mathrm{SH}$ chips were placed in a desiccator with saturated humidity at $40^{\circ} \mathrm{C}$. Images of the chip were captured in each 30 minutes until one of the conditions being completely crosslinked, confirmed by the unchanging of the color intensity. The percentage of reacted amine groups of $\mathrm{CHT}$ for each combination with genipin was quantified by colorimetric assay using the WCIF Image / software. A standard calibration curve (logarithmic curve, $r^{2}=0.98$ ) of pixel color intensity vs. glycine concentration was made. Different quantities of soluble glycine, ranging 0.2 to $2 \mathrm{mg} \mathrm{mL}^{-1}$ were prepared including genipin at a concentration of $10 \mathrm{mg} \mathrm{mL}^{-1}$. The measurements of the pixel intensity from each glycine concentration were performed over a total of 6 samples. The intensity of the pixels, grey scale $0-255$, in which 0 correspond to dark color and 255 white color, was used to associate the color intensity of the solutions. The intensity color of the droplets of $\mathrm{CHT}$ with genipin was correlated with the pixels intensity of the standard curve. The percentage of reacted amine groups in each droplet was determined: the number of reacted amino molecules of the repeating unit of $\mathrm{CHT}$ obtained by the calibration curve was divided by the initial number of amine molecules.

Cell Expansion and Cell Culture: The immortalized mouse lung fibroblast cell line L929 was purchased from the European Collection of Cell Cultures and was used as well established cell line for viability studies. Cells were expanded in low glucose Dulbecco's Modified Eagle's Medium (DMEM), from Sigma-Aldrich, supplemented with $3.7 \mathrm{~g} \mathrm{~L}^{-1}$ sodium bicarbonate, $10 \%$ heat-inactivated fetal bovine serum (FBS, Invitrogen) and 1\% antibiotic/antimycotic (final concentration of penicillin 100 units $\mathrm{mL}^{-1}$ and streptomycin $100 \mathrm{mg} \mathrm{mL}^{-1}$; Gibco) at $\mathrm{pH}$ 7.4. Cells were grown in $75 \mathrm{~cm}^{2}$ tissue culture flasks and incubated at $37{ }^{\circ} \mathrm{C}$ in a humidified air atmosphere of $5 \% \mathrm{CO}_{2}$. Every 3-4 days, fresh medium was added. At $90 \%$ of confluence, L929 cells grown in tissue culture flasks were washed with PBS and subsequently detached by a chemical procedure with $0.05 \%$ trypsin-EDTA solution for 5 min at $37^{\circ} \mathrm{C}$ in a humidified air atmosphere of $5 \% \mathrm{CO}_{2}$. To inactivate the trypsin effect, cell culture medium was added.

On-Chip Biological Testing: Combinatorial analysis of fibroblasts-like cells on patterned SH surfaces was performed. Cells were re-suspended in culture medium with different $\mathrm{pHs}(\mathrm{pH}$ ranging from 1-14) adjusted with $\mathrm{HCl}$ and $\mathrm{NaOH}$ solutions. Droplets of $5 \mu \mathrm{L}$ with $1 \times 10^{3}$ cells were seeded in the micro-indentations of patterned SH surfaces. Specimens were incubated at different times $(5,30,60,90$, and 120 minutes) at $37{ }^{\circ} \mathrm{C}$ in a humidified $5 \% \mathrm{CO}_{2}$ atmosphere. The cell viability was evaluated by a non-destructive live/dead assay. $2 \mu \mathrm{L}$ of phosphate buffer saline PBS (Sigma-Aldrich) containing calcein-AM (1:500) and propidium iodide $(1: 1000)$ was placed at each droplet. Calcein-AM and propidium iodide dyes were purchased from Molecular Probes-Invitrogen. Samples were then incubated at $37{ }^{\circ} \mathrm{C}$ for $10 \mathrm{~min}$ and protected from the light. Droplets were visualized in the dark by fluorescence microscopy (Axioimage RZ1M, Zeiss). Living cells appeared in green and dead cells in red.

3D Hanging Droplet System: A hanging drops system used for the formation of spheroids was also performed using $\mathrm{SH}$ surfaces patterned with adhesive micro-indentations. Prior to cell seeding, the $\mathrm{SH}$ surfaces were sterilized by UV radiation for $30 \mathrm{~min}$. Suspensions with different fibroblasts-like cell densities $\left(6 \times 10^{6}\right.$ and $8 \times 10^{6}$ cells- $\left.\mathrm{mL}^{-1}\right)$ were prepared. Droplets of $5 \mu \mathrm{L}$ of each cell suspensions (in triplicate) were placed over the micro-indentations of the platform. Then, the platform was inverted $180^{\circ}$ and incubated for $24 \mathrm{~h}$ at $37^{\circ} \mathrm{C}$ in a humidified $5 \%$ $\mathrm{CO}_{2}$ atmosphere. The platform containing droplets of cell suspensions was fixed into the lids of polystyrene petri dishes, and the bottom part was filled with cell culture media, in order to create a saturated environment and avoid the evaporation of the droplets. After $24 \mathrm{~h}$ of cell culture for spheroids formation, $2 \mu \mathrm{L}$ of doxorubicin (Doxorubicin hydrochloride, Sigma-Aldrich) with different concentrations $\left(0.1 \mathrm{mg}-\mathrm{mL}^{-1}, 0.01 \mathrm{mg} \mathrm{mL}^{-1}\right.$ and $0.001 \mathrm{mg} \mathrm{mL}^{-1}$ ) were dispensed in the different droplets. The chip was again incubated for $24 \mathrm{~h}$ at $37^{\circ} \mathrm{C}$ in a humidified $5 \% \mathrm{CO}_{2}$ atmosphere. The liquid content of the droplets with cell aggregates were carefully exchange with PBS. Afterwards, live/dead assay was performed. $2 \mu \mathrm{L}$ of PBS containing calcein-AM and propidium was added to each condition to staining the spheroids. Pictures of the formed aggregates were taken in the confocal microscope to provide an accurate quantification (Olympus FluoView FV 1000).

Cell Behaviour Analysis: Image analysis tool (WCIF Image I software) was used to evaluate the percentage of live/dead cells of each droplet condition. Each image of stained cells with culture medium with different $\mathrm{pHs}$ obtained from fluorescent microscope was divided in green and red channels and transformed in a gray scale. The same procedure was made for each image of stained spheroid obtained from confocal microscope. The number in each channel (grey scale intensity $0-255$, in which 0 correspond to black colour and 255 white colour) was counted and divided for the number of total analysed area $\left(300 \times 300\right.$ pixels $\left.^{2}\right)$. The same threshold criterion was used in all images.

\section{Supporting Information}

Supporting Information is available from the Wiley Online Library or from the author. Video 1 shows the possibility to create an active mixing approach based on magnetic microspheres incorporated inside microliter-size droplets. Video 2 shows the dissolution of a crystal in a water droplet with magnetic beads inside controlled by an external mechanical stirrer.

\section{Acknowledgements}

The authors acknowledge the financial support from the FCT- Fundação para a Ciência e para a Tecnologia through the Ph.D. grants with the references SFRH/BD/73119/2010, SFRH/BD/69529/2010 and SFRH/ $\mathrm{BD} / 61390 / 2009$. We also acknowledge the financial support of FEDER through the program Operacional Factores de Competitividade COMPETE and from FCT - the Fundação para a Ciência e para a Tecnologia under the project PTDC/CTM-Bio/1814/2012.

Received: February 13, 2014 Revised: March 24, 2014 Published online:

[1] C. G. Simon, Jr., S. Lin-Gibson, Adv. Mater. 2011, 23, 369.

[2] M. R. Zonca, Jr., P. S. Yune, C. L. Heldt, G. Belfort, Y. Xie, Macromol, Biosci. 2013, 13, 177.

[3] S. W. Cranford, J. de Boer, C. van Blitterswijk, M. J. Buehler, Adv. Mater. 2013, 25, 802.

[4] M. Schenone, V. Dancik, B. K. Wagner, P. A. Clemons, Nat. Chem. Biol. 2013, 9, 232

[5] A. L. Hook, D. G. Anderson, R. Langer, P. Williams, M. C. Davies, M. R. Alexander, Biomaterials 2010, 31, 187.

[6] A. N. Efremov, E. Stanganello, A. Welle, S. Scholpp, P. A. Levkin, Biomaterials 2013, 34, 1757.

[7] D. M. Titmarsh, H. Y. Chen, E. J. Wolvetang, J. J. Cooper-White, Biotechnol. J. 2013, 8, 167.

[8] S. A. Sundberg, Curr. Opin. Biotechnol. 2000, 11, 47.

[9] Y. C. Tung, A. Y. Hsiao, S. G. Allen, Y. S. Torisawa, M. Ho, S. Takayama, Analyst 2011, 136, 473

[10] C. Timm, C. M. Niemeyer, Angew. Chem. Int. Ed. Engl. 2013, 52, 2652.

[11] Y. M. Foong, J. Fu, S. Q. Yao, M. Uttamchandani, Curr. Opin. Chem. Biol. 2012, 16, 234.

[12] G. M. Luz, Á. J. Leite, A. I. Neto, W. Song, J. F. Mano, Mater. Lett. $2011,65,296$ 
[13] A. I. Neto, C. A. Custódio, W. Song, J. F. Mano, Soft Matter 2011, 7, 4147.

[14] C. L. Salgado, M. B. Oliveira, J. F. Mano, Integr. Biol. 2012, 4, 318.

[15] M. B. Oliveira, J. F. Mano, Anal. Chem. 2013, 85, 2391.

[16] M. B. Oliveira, C. L. Salgado, W. Song, J. F. Mano, Small 2013, 9, 768.

[17] N. M. Oliveira, A. I. Neto, W. Song, J. F. Mano, Appl. Phys. Exp. 2010, 3, 085205

[18] S. M. Oliveira, W. L. Song, N. M. Alves, J. F. Mano, Soft Matter 2011, 7, 8932.

[19] A. I. Neto, H. J. Meredith, C. L. Jenkins, J. J. Wilker, J. F. Mano, RSC Adv. 2013, 3, 9352

[20] X. Hong, X. F. Gao, L. Jiang, J. Am. Chem. Soc. 2007, 129, 1478.

[21] L. Feng, Y. A. Zhang, J. M. Xi, Y. Zhu, N. Wang, F. Xia, L. Jiang, Langmuir 2008, 24, 4114.

[22] Y. Y. Yan, N. Gao, W. Barthlott, Adv. Colloid Interface Sci. 2011, 169, 80.

[23] M. Nosonovsky, B. Bhushan, Green Tribology 2012, 25.

[24] Z. Cheng, M. Du, H. Lai, N. Zhang, K. Sun, Nanoscale 2013, 5, 2776.

[25] H. Song, D. L. Chen, R. F. Ismagilov, Angew. Chem. Int. Ed. Engl. 2006, 45, 7336.

[26] S. Y. Teh, R. Lin, L. H. Hung, A. P. Lee, Lab Chip 2008, 8, 198

[27] B. Su, S. Wang, Y. Song, L. Jiang, Nano Res. 2010, 4, 266.

[28] M. A. M. Gijs, F. Lacharme, U. Lehmann, Chem. Rev. 2010, 110, 1518.

[29] A. Accardo, F. Mecarini, M. Leoncini, F. Brandi, E. Di Cola, M. Burghammer, C. Riekel, E. Di Fabrizio, Lab Chip 2013, 13, 332.

[30] Z. Long, A. M. Shetty, M. J. Solomon, R. G. Larson, Lab Chip 2009, 9, 1567.

[31] D. De Bruyker, M. I. Recht, A. A. Bhagat, F. E. Torres, A. G. Bell, R. H. Bruce, Lab Chip 2011, 11, 3313.

[32] M. Shikida, K. Takayanagi, K. Inouchi, H. Honda, K. Sato, Sens. Actuat. B 2006, 113, 563.

[33] J. Schneider, A. Egatz-Gómez, S. Melle, S. Lindsay, P. DomínguezGarcía, M. A. Rubio, M. Márquez, A. A. García, Colloids Surf., A 2008, 323, 19.

[34] J. S. Sander, R. M. Erb, C. Denier, A. R. Studart, Adv. Mater. 2012, 24, 2582.

[35] F. Sarvi, Z. Yue, K. Hourigan, M. C. Thompson, P. P. Y. Chan, J. Mater. Chem. B 2013, 1, 987.

[36] J. L. Drury, D. J. Mooney, Biomaterials 2003, 24, 4337.

[37] A. Khademhosseini, R. Langer, Biomaterials 2007, 28, 5087.

[38] D. Seliktar, Science 2012, 336, 1124.

[39] J. K. F. Suh, H. W. T. Matthew, Biomaterials 2000, 21, 2589.
[40] J. F. Mano, G. A. Silva, H. S. Azevedo, P. B. Malafaya, R. A. Sousa, S. S. Silva, L. F. Boesel, J. M. Oliveira, T. C. Santos, A. P. Marques, N. M. Neves, R. L. Reis, J. R. Soc. Interface 2007, 4, 999.

[41] N. M. Alves, J. F. Mano, Int. J. Biol. Macromol. 2008, 43, 401.

[42] S. S. Silva, A. Motta, M. T. Rodrigues, A. F. M. Pinheiro, M. E. Gomes, J. F. Mano, R. L. Reis, C. Migliaresi, Biomacromolecules 2008, 9, 2764.

[43] R. A. A. Muzzarelli, Carbohydr. Polym. 2009, 77, 1.

[44] L. P. Yan, Y. J. Wang, L. Ren, G. Wu, S. G. Caridade, J. B. Fan, L. Y. Wang, P. H. Ji, J. M. Oliveira, J. T. Oliveira, J. F. Mano, R. L. Reis, J. Biomed. Mater. Res., Part A 2010, 95A, 465.

[45] M. Arteche Pujana, L. Perez-Alvarez, L. C. Cesteros Iturbe, I. Katime, Carbohydr. Polym. 2013, 94, 836.

[46] F. L. Mi, Y. C. Tan, H. F. Liang, H. W. Sung, Biomaterials 2002, 23, 181.

[47] T. G. Fernandes, M. M. Diogo, D. S. Clark, J. S. Dordick, J. M. Cabral, Trends Biotechnol. 2009, 27, 342.

[48] X. X. Zhang, Z. Wang, X. Yue, Y. Ma, D. O. Kiesewetter, X. Chen, Mol. Pharm. 2013, 10, 1910.

[49] A. Hulikova, A. L. Harris, R. D. Vaughan-Jones, P. Swietach, J. Cell Physiol. 2013, 228, 743.

[50] T. M. Cao, T. Takatani, M. R. King, Biophys. J. 2013, 104, 292.

[51] D. R. Knighton, T. K. Hunt, H. Scheuenstuhl, B. J. Halliday, Z. Werb, M. J. Banda, Science 1983, 221, 1283.

[52] Y. Bae, S. Fukushima, A. Harada, K. Kataoka, Angew. Chem. Int. Ed. Engl. 2003, 42, 4640.

[53] Y. Lu, J. J. Chen, L. Mu, Q. Xue, Y. Wu, P. H. Wu, J. Li, A. O. Vortmeyer, K. Miller-Jensen, D. Wirtz, R. Fan, Anal. Chem. 2013, 85, 2548.

[54] J. Zhou, C. Fang, T. Chang, X. Liu, D. Shangguan, J. Mater. Chem. B 2013, 1,661.

[55] J. Kononen, L. Bubendorf, A. Kallioniemi, M. Barlund, P. Schraml, S. Leighton, J. Torhorst, M. J. Mihatsch, G. Sauter, O. P. Kallioniemi, Nat. Med. 1998, 4, 844 .

[56] Z. Xu, Y. Gao, Y. Hao, E. Li, Y. Wang, J. Zhang, W. Wang, Z. Gao, Q. Wang, Biomaterials 2013, 34, 4109.

[57] G. Cheng, J. Tse, R. K. Jain, L. L. Munn, PLoS One 2009, 4, e4632.

[58] A. Y. Hsiao, Y. C. Tung, C. H. Kuo, B. Mosadegh, R. Bedenis, K. J. Pienta, S. Takayama, Biomed. Microdevices 2012, 14, 313.

[59] J. C. Schulz, P. S. Stumpf, A. Katsen-Globa, A. Sachinidis, J. Hescheler, H. Zimmermann, Eng. Life Sci. 2012, 12, 584.

[60] E. Fennema, N. Rivron, J. Rouwkema, C. van Blitterswijk, J. de Boer, Trends Biotechnol. 2013, 31, 108.

[61] Y. Morimoto, S. Takeuchi, Biomater. Sci. 2013, 1, 257. 